\title{
Using Adaptive Architecture to Support Yoga Practices: Social Considerations for Design
}

\author{
Nils Jäger ${ }^{1}$, Stuart Moran ${ }^{2}$, Holger Schnädelbach ${ }^{3}$ \\ Mixed Reality Lab \\ University of Nottingham, UK \\ 1'psxnj@nottingham.ac.uk, ${ }^{2,3}$ [firstname.lastname]@nottingham.ac.uk
}

\begin{abstract}
The field of Adaptive Architecture aims to design built environments, which truly adapt to their occupants. ExoBuilding is an in-house prototypical example of Adaptive Architecture, which actuates in response to breathing and heartrate of its occupants. In this work-in-progress paper, we discuss our aims to apply the technology to the practice of Yoga, in which a core aspect is controlled breathing. We explore the social considerations of deploying this novel technology, and then examine the different possibilities for interaction.
\end{abstract}

Keywords-Adaptive Architecture; Yoga; ExoBuilding; Breathing; Physiological; Teaching;

\section{INTRODUCTION}

The field of Adaptive Architecture, concerned with buildings that are specifically designed to adapt to inhabitants and the environment, is developing rapidly [1]. Multiple research strands across the fields of computer science, architecture, engineering and the social sciences contribute to our understanding of this multi-disciplinary field [2]. The specific buildings that have emerged have been proposed for example to increase energy efficiency, to modulate inhabitant comfort, to support organizational flexibility and in support of particular activities. One recurring interest in the field of Adaptive Architecture is concerned with the role that personal data can play in this space [3].

Technically and principally, personal data can be made available to our surroundings through ubiquitous computing technologies, whether this is within a building or in urban spaces. Across the field of Adaptive Architecture this has already been explored through location data, physiological data or social networking data [3]. Recent work with ExoBuilding, an exemplar piece of Adaptive Architecture, which maps physiological data to its form, has demonstrated the emerging feedback loop that occurs by mapping personal information to the built environment (see Section II). Personal data mapped to actuations in an immersing environment feeds back on inhabitants, who change their behaviour in turn [4].

Arguably, some of the most innovative, recent work in the field of Adaptive Architecture so far lacks footing in real world applications. Links between personal data and actuations in the built environment have often been explored to address conceptual questions, and they have been technology or design driven. Many of the results are highly playful and also engaging, while they are not studied in much detail or for any length of time.

The emerging research programme around ExoBuilding, which this paper is a part of, addresses the above in two different ways. It carefully investigates a number of related research questions over time through lab-based investigations. The other strand of the work is to actively pursue real world applications of the knowledge gathered so far, with a view to deploy technology and study its use 'in the Wild'; in this case, our initial exploration is into the practices of Yoga. In what follows, we will start to address this latter concern beginning with an overview of the ExoBuilding prototype Adaptive Architecture as it currently stands.

\section{EXOBUILDING}

ExoBuilding is a prototype Adaptive Architecture; and more specifically, it is an instance of Biofeedback Architecture. As Brand argued in "How Buildings Learn" [5], all architecture is inherently adaptable given the right tools and enough time. Adaptive Architecture, as opposed to conventional architecture, consists of structures specifically designed to adapt or respond to environmental changes, such as climate, lighting conditions, or, as pertinent to ExoBuilding, human behaviour.

Adaptive architecture often incorporates physical alterations of central architectural features, sometimes even structural elements. These alterations can be analogue or digital, which has been explained in more detail by Schnädelbach [1]. Here, we are concerned with digitallydriven architecture as described by Bier and Knight [6]. Examples of this include Muscle Tower [7], Open Columns [3], and Interactive Wall [8], all cases in which the structure of the architecture itself adapts based on sensed properties of the environment. Muscle Tower uses muscle wires to twist and bend or lean towards passers-by, thereby assuming a life-like character. Open Columns, consisting of ceiling-mounted composite urethane elastomer mesh columns, react to increases in $\mathrm{CO}_{2}$ levels of the interior space and lower themselves to the floor. This lowering action disperses crowds, who are the original cause of increased $\mathrm{CO}_{2}$ levels, and changes the spatial configuration of the room, forcing inhabitants to change their physical and social occupancy of the space.

Similar to Muscle Tower, the conically shaped wall segments of Interactive Wall detect proximity of humans and sway in response creating undulating motion patterns. As these examples show, the common design principle of Adaptive Architecture projects is to create architecture with adaptation in mind from the first design stages. This sets Adaptive Architecture apart from Smart Homes, which can consist of traditionally designed architecture, which is then 

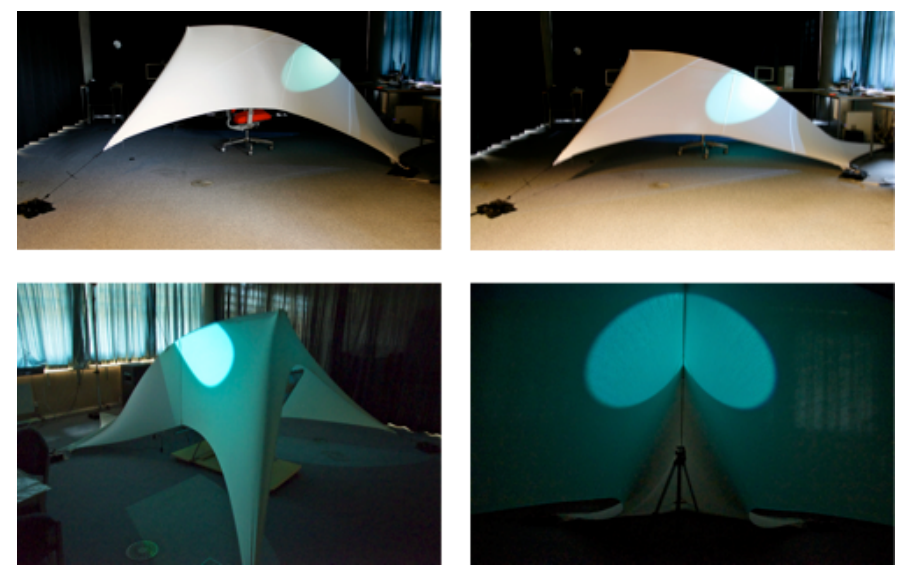

Figure 1 ExoBuilding prototype Adaptive Architecture. Top row (left to right): up state, down state. Bottom row (left to right): back view, inhabitant view.

augmented with technology, typically without the intention to physically or structurally alter key architectural elements or spatial configurations. As evident from Chan's [9] review paper on Smart Homes, the focus here is on the inhabitant's comfort, convenience, or efficiency.

Although Muscle Tower, Open Columns, and Interactive Wall respond to humans and their behaviour, they do not explicitly adapt to physiological processes, unlike ExoBuilding. As an example of Biofeedback Architecture, a subset of Adaptive Architecture, ExoBuilding reacts to its inhabitant's physiological processes. Thus, Biofeedback Architecture consists of architectural structures that sense an inhabitant's physiological behaviour, such as respiration (RSP) or heart rate (HR), and reflect this behaviour through spatial actuations in real-time. We envision to eventually give ExoBuilding the ability to create client profiles. This would enable it to learn over time how each client behaves and how ExoBuilding should tailor its own behaviour to achieve the most beneficial effects for each client.

Specifically, ExoBuilding is a single-inhabitant, tent-like structure that changes its height, volume, and shape, as well as colour and sound based on its inhabitant's real-time physiological data. Recently, colour and sound adaptations were disabled to avoid confounding effects during the experimental studies. Jersey fabric stretches over an aluminium spine, which itself is attached to two ceilingmounted servomotors. The motors allow the spine to be vertically raised by about 30 centimetres between the up and down state (Figure 1). Combining physical structure, biosensing technology (MindMedia NeXus-10 and BioTrace+ www.mindmedia.info/) and a custom middleware platform (ECT) [10] allows direct physiological interaction between inhabitant and environment. While inhabited, ExoBuilding is mainly a dark environment as the ceiling lights are extinct and only the projection of a circular blue graphic and residual light remains visible (Figure 1).

Originally, ExoBuilding developed out of the research question whether physiological data could relate to architecture, and if so in which ways [11][4]. The approach was to make apparent generally hard-to-see or invisible physiological processes of an inhabitant through the inhabited building. The externalisation of physiological processes, then, results in the building supporting the inhabitant's physiological behaviours, similar to an exoskeleton. Currently, we mainly map respiration and heart rate to the building's motion. In a respiration mapping, for example, when an inhabitant inhales, the servomotors pull up the aluminium spine in real-time, thereby increasing the volume of the building, changing its shape through the slight flex of the metal extrusion, as well as making the fabric billow noticeably. The subsequent exhalation of the inhabitant causes the servomotors to rotate in the opposite direction, lowering the spine down again.

So far, we have conducted three formal studies investigating the inhabitation of biofeedback architecture. The first study showed that respiratory biofeedback through ExoBuilding caused un-instructed participants to breathe slower, deeper, and more regularly compared to a no-motion and a regular-motion (automated motion) condition [12]. In a second study (under review), participants experienced heart rate biofeedback through ExoBuilding. However, a computer agent severed the physiological link between participant and environment in order to attempt to manipulate participant behavior. The aim was to make the participant breath twenty per cent slower after a certain amount of time. From the participant's perspective, the severance of the physiological link to the environment can be described as an effective loss of control over the environment. Most participants did indeed reduce their RSP frequency according to the manipulation. A third study (also under review) compared an immersive with a non-immersive condition. Participants reported greater liking of the immersive condition and expressed greater ease in breathing regularly (as instructed), which allowed some to fall into states of meditation and/or deep relaxation.

In summary, our studies indicate that biofeedback through ExoBuilding allows inhabitants to fall into sustainable, regular and self-regulating respiratory patterns with (or without) minimal instructions. Next, we started to explore potential 'real-world' application domains. For example, we envision an application of biofeedback architecture in health-related technology or environments, such as rehabilitation environments. Additionally, we can conceive of applying this technology to leisure environments reminiscent of saunas or flotation tanks, environments that clients in search of relaxation can frequent on occasional or regular bases.

Since we employ respiration as one of our main physiological behaviours to actuate the environment, we sought out collaboration with an experienced yoga teacher. The teacher expressed interest in exploring ExoBuilding in relation to the practice of yoga, for example as a teaching tool and a collaborative environment.

\section{YOGA}

Yoga, meaning "union" or "joining", is the practice of balance and unity of the body and mind [13]. The overarching goal is to reach a sense of union between the self and the world, 
resulting in inner peace or awareness. This is typically achieved through practice of a variety of physical and mental exercises. These include a range of physical postures called asanas, including standing, seated, lying, bends, and twists. Breathing patterns, exercises and regulation are also a vital part of the practice. Breathing plays an important role in almost every aspect of Yoga, with a general rule of thumb: $50 \%$ of concentration should always be $100 \%$ on breathing. When controlled breathing is combined with asanas, they form vinyasas, or yoga flows, which see synchronized movement and breathing. There are many empirically proven health benefits from practicing yoga, including improvements related to heart rate variability [14], blood glucose [15], fatigue [16], and depression [17].

Yoga is frequently taught and practiced in a group environment [18]. This can create a shared social learning experience [18], [19] and sense of group mindfulness [18], [20], [21]. It can also form a peer network which can support the activity and socially sustain motivation [18], [19]. Although taught in a group, yoga is predominately an individual pursuit [19]. Not only are there significant physical differences between individual bodies, but there are also gaps in the skill and duration with which yoga students execute each asana [19]. For this reason, a teacher must carefully consider each individual's abilities [18], and actively discourage competitiveness due to risk of over exertion [19].

\section{A. Technology Mediated Yoga}

Practicing yoga often requires the use of props such as blankets, blocks, straps and chairs, to support alignment and perform deeper asanas. These props have been described as mediators with the aim of providing feedback, creating awareness and connections between parts of the body [13].

Some research laboratories and industry companies have recently developed computing technologies with a focus on exclusively mediating yoga practices [18]. For example, the 'Therapeutic Interactive Yoga' is an immersive space, which tracks the user's body, and responds with different colours, sounds, and even scents appropriate to each asana. Similarly, the 'Interactive Yoga Studio' [18] is a space comprised of an interactive yoga matt, projected visualizations and a surround sound audio accompaniment to create a relaxing environment for daily practitioners. 'Yoga Social Mats' [22] are augmented with embedded sensors to detect activity and employ networked display tablets used to connect users socially.

In terms of commercial products, the video games industry has driven the development of novel controller technology designed to support yoga practices. The Nintendo Wii-Balance Board, which is similar to an electronic weighing scale, sensitively measures the pressure the user applies to it. The board then assesses the user's balance and posture and provides visual and auditory feedback. This technology has even been used to support and teach breathing in seated postures [23]. In contrast, Microsoft's Kinect uses a camera and computer vision techniques to model and track users' limbs and joints in real-time. By comparing the shape of the user's posture to an established 'best-practice', users can receive auditory and visual feedback. This technology has
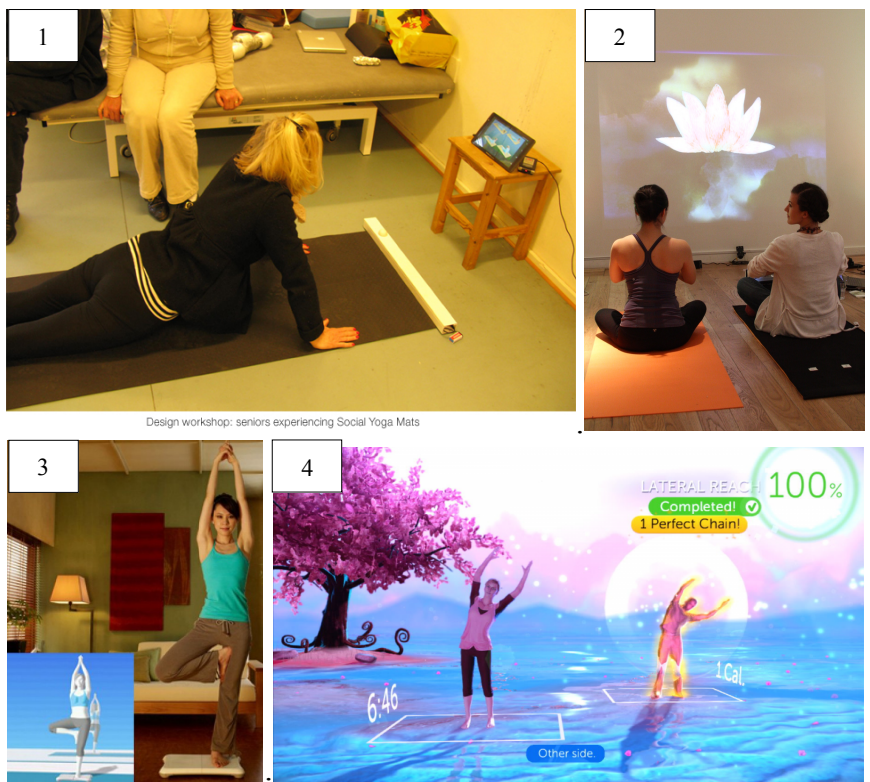

Figure 2 Technology mediated yoga, including (1) Social Yoga Matts, (2) Interactive Yoga Project, (3) Nintendo Wii-Fit and (4) Microsoft Kinect with YourShape:Fitness Evolved.

been used to assist the partially-sighted and blind practice yoga [24]. As a result of augmenting Yoga with technology, virtually immersive environments [18], increased levels of personal relaxation [25], increased group awareness [19], [22], and multisensory feedback and teaching support [25] have emerged. Even with such advancements, there is still further need for more direct technological support for yoga, including features such as better instruction, monitoring and feedback [22], and more accurate assessment on exercises [19].

To date, the majority of this technology has focused on collecting data based on what is visible on the 'outside' of the body, rather than what is inside [25]. There are many reasons for this, including the need for real-time feedback and the slow postures making it ideal for gesture recognition [25]. Furthermore, the feedback users receive from these technologies is, for the most part, visual in nature. Hence, we propose to explore a novel approach to technology-mediated yoga. We aim to collect physiological data, representing the unobservable 'inner space' of a person and relay that information back to them in the form of physical actuations of the immediate environment using ExoBuilding, our existing example of adaptive architecture.

\section{ExOBUILDING AND YoGA}

To ground the investigation of ExoBuilding's potential use in Yoga practice we sought out collaboration with a local, wellestablished yoga teacher. In two meetings and one workshop we explored the possibilities of how to integrate ExoBuilding into the practice of yoga and the practice of teaching yoga. This involved some exploratory group exercises lead by the yoga teacher, leading the three authors through a set of respiration exercises conducted in and around ExoBuilding, with one of the four actors' physiological data (respiration) being mapped to prototype actuation. The following is 
drawing on participant feedback gathered through multiple previous studies mentioned above (not focusing on yoga specifically), the initial explorations described above and the long-term experience with yoga that one of the authors brings.

There are several ways we envision ExoBuilding to be used as part of yoga practice. The most obvious is to externalize internal body functions through a spatial and tactile actuation. As ExoBuilding can be driven by multiple sources of biometric measures, there are many different aspects of the user, which can be used to drive the actuations of ExoBuilding. This creates a unique opportunity to support the teaching of Yoga, including breathing exercises and control of heart rate during asanas and meditation, for which we intend to use findings from our previous studies in order to create the desired relationships between yoga student and environment as well as creating beneficial outcomes. By externalizing physiological information, ExoBuilding enables both teacher and learner to objectively share their true physiological, and by extension mental, state. The real-time interaction could also more easily enable users to clearly observe their progress, and have a greater sense of connection with the world. From a teacher's perspective, this would also give a clearer indication of the skill level and state of their student, allowing them to better inform their choice in teaching approaches and advice. Aside of learning support, ExoBuilding itself could augment the environment of practice. Users are anticipated to practice inside ExoBuilding, which may create a more relaxing, private and immersive environment that is configurable to different asanas. This is an important feature, as (virtual) immersion has been shown to be critical when learning to physically coordinate the body [26].

So far we have focused on the individual, but we also envisage ExoBuilding as usable by a group. With group cohesion and awareness a valuable aspect of Yoga classes, ExoBuilding may serve as a means of promoting synergy and motivation. For example, by collectively following a set or user-controlled rhythm through ExoBuilding, students may achieve a synergetic and motivating group experience.

While ExoBuilding is directly useable in its current form, planned additions will increase the possibilities for use. For example, a long-term goal is for multiple users to interact with ExoBuilding at the same time, each contributing their own physiological measures to the spatial actuations displayed. Furthermore, the possibility of introducing software agents or machine learning into ExoBuilding would allow us to tailor the system to each individual's own practice, providing a novel form of teaching. This type of tailoring will create a more profound and meaningful interaction with the technology [25]. From the above, the potential for the use of ExoBuilding in Yoga practices starts to emerge. However, before considering the different possible modes of interaction, we must first consider the potential impact the technology might have on the practice to better inform the design.

\section{SOCIAL CONSIDERATIONS}

To explore the idea of using ExoBuilding to support yoga practices we conducted an initial scoping study, where we observed a series of yoga classes. The attendees of these classes were unaware of the observations, as one of the authors is a regular member of the yoga group. For a series of six classes, he intentionally observed both teacher and students and their interactions. However, some of our questions, and design ideas might have originate from class sessions prior to or after these six sessions. We also ran a series of informal workshops to discuss the concept of using ExoBuilding in connection with yoga with a yoga teacher. Through this process we noted several points that can better inform the design of different modes of interaction with ExoBuilding. These centre on the implications of revealing physiological data to users, the impact of introducing computing technology to the practice, and issues related to trust and data privacy.

\section{A. Quantifying the Self}

One of the major points for consideration is the implication of revealing physiological data to a user. Particularly as physiological data is something most people are unlikely to have ever experienced in a quantified way outside of a healthcare environment. Could such an action or feature empower the teacher, the student, or both? Allowing what is a highly subjective activity to become objective may strengthen the user's yoga practice. In terms of a teacher-student relationship, it could surface the student's true current state and skill set, allowing the student to more effectively communicate and assess their progress. Equally, a teacher could demonstrate what is expected in a specific state more easily, and provide a comparison point with the student. In fact, introducing this level of objectivity alone could lead to more dynamic and stimulating approaches to teaching.

However, there are a number of serious considerations about introducing such objectivity to yoga. In the first case, it might instill a sense of competition both internally for an individual, and even more so within a group. Yoga remains an internal endeavor, and objectively externalizing aspects of human physiology may lead to undesirable competitive effects brought about by the gained ability to compare with one another. This may, in turn, negatively influence the overall group dynamic, and so must be carefully considered during the design process. Additionally, revealing an objective assessment of progress in an individual pursuit may lead to users exerting themselves beyond their own abilities in an attempt to improve in the eyes of others $-\mathrm{a}$ form of negative social facilitation [27].

This should be avoided if possible, and the focus be made on the solidarity brought about through an individual, but shared, practice. We may achieve this goal through careful and balanced design of the modes of interaction with ExoBuilding. There is potential for the technology to support practitioners, and surfacing their physiological data could create 'short-cuts' to learning, and achieving, an enlightened state. It is questionable whether this is a positive outcome, particularly as there are many potential issues surrounding the skill and time typically invested in developing strong self-awareness. The remote possibility for over-reliance on the technology to achieve such states of enlightenment could further exacerbate 
the problem of developing the necessary skills to achieve the desired self-awareness.

\section{B. Disrupting the Inner Peace}

Given the focus on the body and the mind, yoga is not an activity readily associated with the use of technology. For many it is a journey into the self, and an escape from technology. Hence, the benefits of introducing any technology to the practice should be carefully weighed against any negative impact. For example, one of the major practical limitations of ExoBuilding is the current need to attach fullcontact sensors to the participants' bodies. Wearing such attachments throughout a session, or interrupting a practice to put them on, are likely to become a disruption. This may be manageable during a session with a single student, but is likely to be problematic for groups. However, if the teacher wore the technology and controlled ExoBuilding, the disruption of the practice session caused by attaching sensors to members of the group might be offset; particularly given that the teacher frequently takes breaks during a practice to help students adjust and align to specific asanas. Finally, as the technology is still in its prototypical state, malfunctions are likely to be expected. This could range from miss-alignment with a user's physiological input to a lag in communication between devices to a complete breakdown of software, hardware, or both. Any such malfunction would likely disrupt the practice of yoga, and so we need a simple exit strategy for the technology to minimize its impact. Protecting the health and safety of those using the technology is paramount.

\section{Trust and Privacy}

Collecting and revealing physiological data is a unique experience for most people, with the expected context for this type of monitoring in a healthcare environment. Revealing such a private and hidden part of ourselves to others requires a strong level of trust. Trust is an important factor both in terms of how practitioners use the technology and who uses it, and of the trajectory of the information collected over time. Users cannot easily mask the actuations caused by their physiological data, and hence must feel open to sharing their data. Luckily, this act of sharing is already common in Yoga, with the need to be open and honest about any difficulties.

A strong ethical consideration is that in some cases, physiological data can reveal a health problem that may be unbeknownst to the user. Should those who notice it reveal such information? It may cause unnecessary panic, but equally, if left unchecked could lead to more serious problems. Furthermore, what of a situation where the data presented is incorrect due to limitations in the technology? There are also questions surrounding the implications of storing the data, and how it might be used in the future, in addition to the length and period of recording of user information. Much of these issues of ExoBuilding also apply to other contexts, and have been explored in more detail in previous research [28].

\section{INFORMING SYSTEM DESIGN}

With the above initial observations regarding the potential implications of introducing this technology and its usage in mind, we examined a number of envisaged modes of interaction with ExoBuilding.

\section{A. Individuals and Groups}

We propose designing a variety of different exercises with ExoBuilding in mind, satisfying both group and individual needs. We imagine exercises where the group follows either an automated pattern or the teacher in unison with the possibility of switching input - control over ExoBuilding between individuals. This will retain the need for group cohesion and awareness, while acknowledging that each individual is different and the desire for a tailored experience.

\section{B. The Driver}

With different arrangements for groups and individuals, it is necessary to consider the different ways of driving ExoBuilding. For example, when teaching an individual it might make sense for the student to drive, and the teacher to observe. On the other hand, a teacher, when controlling ExoBuilding, might be able to demonstrate specific breathing patterns and frequencies more effectively than through verbal expression. While in a group situation, it might be most efficient to have a teacher control the environment for the same reason. Preset patterns run by the software are yet another option to structure a session. Equally, in both individual and group instances, it may be useful to pass control between different people randomly, in a preset order, or through a token.

\section{Sharing}

As mentioned earlier, privacy and trust are key factors when dealing with the sharing of physiological data. One way of protecting users of the environment could be to mask or filter the data in some way, so as to not reveal everything that is observable, and only use what is needed to drive the environment. It may be interesting to explore how multiple inputs could be aggregated and fused together, not only to mask the data from individuals, but also to create a true sense of group cohesiveness with everyone understanding that they are contributing to the driving of the building. Ultimately, we are able to give users complete control of ExoBuilding with regard to the time and fashion of the use of their data in realtime, as well as storage of data.

\section{Immersion}

As our studies have shown, immersion is one of the key elements of ExoBuilding's effectiveness. The degree of immersion or immersive-ness might vary depending on the manner of installation of ExoBuilding. For example, depending on spatial constraints of a given room, ExoBuilding, instead of being ceiling-hung, could be mounted sideways to a wall or inverted (upside down). The size of the environment is also likely to bare influence on the degree of immersion in addition to the numbers of points of actuation. Further to the potential limitations regarding research in a yoga setting, we need to consider and anticipate challenges posed by the physical environment in which ExoBuilding might be installed, such as restrictions regarding the size and 
shape of ExoBuilding. It is also possible that the physical setting does not allow a ceiling-mounted version of ExoBuilding, which would require a fundamental re-design of this biofeedback environment. Furthermore, the prototypical nature of both software and hardware might prove unfeasible and possibly inoperable in a yoga class setting due to its fragility. We should also be prepared for clients to reject any form of technology in their class sessions or object to the obtrusiveness of the body-worn sensors

\section{FUTURE WORK}

Alongside the exploration of ExoBuilding in a yoga setting, we are currently developing a multi-user environment that will allow us to study interactions between multiple inhabitants, be it in a teaching or non-teaching setting. Specifically, we are interested in the dynamics of interaction between inhabitants as well as between inhabitants and the new environment. As mentioned above, an interesting line of investigation will be the handling of multiple live and automated data streams controlling the environment. We are currently preparing a series of studies designed to explore the ways in which ExoBuilding can be used to teach Yoga, in addition to its potential for creating and sustaining group cohesion.

\section{CONCLUSIONS}

We described the current state of research pertaining to a prototype of Biofeedback Architecture called ExoBuilding and our intentions to expand our research to studies of the technology in a real-world setting. We showed that ExoBuilding as an instance of Biofeedback Architecture lends itself to application in yoga settings. It offers opportunities for innovative and objectified teaching methods of yoga, albeit with the expressed concerns regarding data privacy and the potential introduction of undesired competition.

\section{REFERENCES}

[1] H. Schnädelbach, "Adaptive Architecture - A Conceptual Framework," in Proceedings of MediaCity, 2010.

[2] L. Bullivant and $\mathrm{H}$. Castle, 4dspace: interactive architecture. WileyAcademy, 2005.

[3] O. Khan, "Open columns: a carbon dioxide (CO2) responsive architecture.," in In CHI'10 Extended Abstracts on Human Factors in Computing Systems, 2010, pp. 4789-4792.

[4] H. Schnädelbach, "Physiological Data in Adaptive Architecture," in International Adaptive Architecture Conference, , 2011, pp. 1-15.

[5] S. Brand, How buildings learn: what happens after they're built. Penguin, 1995.

[6] Bier, H. and T. Knight, Digitally-Driven Architecture. Delft: 6th Footprint Issue, Stichting Footprint, 2010, pp. 1-4.

[7] K. Oosterhuis and N. Biloria, "Interactions with proactive architectural spaces: the muscle projects," Commun. $A C M$, vol. 51 , no. 6, pp. 70-78, 2008.

[8] M. Hosale and C. Kievid, "Modulating territories, penetrating boundaries," in in Digitally-Driven Architecture, Delft: 6th Footprint Issue, Stichting Footprint, 2010, pp. 55-67.

[9] M. Chan, D. Estève, C. Escriba, and E. Campo, "A review of smart homes-Present state and future challenges," Computer methods and programs in biomedicine, vol. 91, no. 1, pp. 55-81, 2008.

[10] S. Rennick-Egglestone, J. Humble, C. Greenhalgh, T. Rodden, and A. Hampshire, "The Equator Component Toolkit: Managing digital information flow in the home," in Adj. Proc. of UIST, 2006, vol. Egglestone.

[11] H. Schnädelbach, K. Glover, and A. A. Irune, "ExoBuilding: breathing life into architecture," in Proceedings of the 6th Nordic Conference on Human-Computer Interaction: Extending Boundaries, 2010, pp. 442451.

[12] H. Schnädelbach, A. Irune, D.Kirk, K.Glover, and P.Brundell, "ExoBuilding: Physiologically Driven Adaptive Architecture," TOCHI, vol. 19, no. 4, p. 25, 2012.

[13] J. Larssen, A. T., Robertson, T., \& Edwards, "The feel dimension of technology interaction: exploring tangibles through movement and touch.," in roceedings of the 1st international conference on Tangible and embedded interaction, 2007, pp. 271-278.

[14] A. J. Bowman, R. H. Clayton, A. Murray, J. W. Reed, M. M. F. Subhan, and G. A. Ford, "Effects of aerobic exercise training and yoga on the baroreflex in healthy elderly persons," European journal of clinical investigation, vol. 27, no. 5, pp. 443-449, 1997.

[15] S. Sinha, S. N. Singh, Y. P. Monga, and U. S. Ray, "Improvement of glutathione and total antioxidant status with yoga," The Journal of Alternative and Complementary Medicine, vol. 13, no. 10, pp. 10851090, 2007.

[16] B. S. Oken, S. Kishiyama, D. Zajdel, D. Bourdette, J. Carlsen, M. Haas, C. Hugos, D. F. Kraemer, J. Lawrence, and M. Mass, "Randomized controlled trial of yoga and exercise in multiple sclerosis," Neurology, vol. 51, no. 11, pp. 2058-2064, 2004.

[17] D. Shapiro, I. A. Cook, D. M. Davydov, C. Ottaviani, A. F. Leuchter, and M. Abrams, "Yoga as a complementary treatment of depression: effects of traits and moods on treatment outcome," Evidence-based complementary and alternative medicine, vol. 4, no. 4, pp. 493-502, 2007.

[18] M. Park, M. Pinto, M.-C. Chen, and A. Gomes, "Enhancing the Experience of Yoga Practice through an Immersive Interactive Studio," in APCHI 2012, August 28-31, 2012, Matsue, Japan, 2012.

[19] T. Nagargoje, A., Maybach, K., \& Sokoler, "Social yoga mats: designing for exercising/socializing synergy," in In Proceedings of the Sixth International Conference on Tangible, Embedded and Embodied Interaction, 2012, pp. 87-90.

[20] J. P. Shelov, D. V., Suchday, S., \& Friedberg, "A pilot study measuring the impact of yoga on the trait of mindfulness," Behavioural and cognitive psychotherapy, vol. 37, no. 5, p. 595, 2009.

[21] N. Chadwick, P., Taylor, K. N., \& Abba, "Mindfulness groups for people with psychosis," Behavioural and Cognitive Psychotherapy, vol. 33, no. 3, p. 351, 2005.

[22] T. Maybach, K., Nagargoje, A., \& Sokoler, "Social yoga mats: reinforcing synergy between physical and social activity.," in CHI'll Extended Abstracts on Human Factors in Computing Systems, 2011, pp. $1561-1566$

[23] J. F. Esculier, J. Vaudrin, P. Beriault, K. Gagnon, and L. E. Tremblay, "Home-based balance training programme using wii fit with balance board for parkinson's disease: A pilot study," Journal of Rehabilitation Medicine, vol. 44, no. 2, pp. 144-150, 2012.

[24] J. A. Rector, K., Bennett, C. L., \& Kientz, "Eyes-free yoga: an exergame using depth cameras for blind \& low vision exercise," in In Proceedings of the 15th International ACM SIGACCESS Conference on Computers and Accessibility, 2013, p. 12.

[25] P. Fels, S., Gauthier, J., \& Smith, "Responses in light, sound and scent: a therapeutic interactive yoga system," in In IWEC, 2002, pp. 355-362.

[26] R. Patel, K., Bailenson, J. N., Hack-Jung, S., Diankov, R., \& Bajcsy, "The effects of fully immersive virtual reality on the learning of physical," in In Proceedings of the 9th Annual International Workshop on Presence, 2006, p. 87-.

[27] B. Strauss, "Social facilitation in motor tasks: a review of research and theory," Psychology of Sport and Exercise, vol. 3, pp. 237-256, 2001.

[28] S. Moran, N. Jaeger, H. Schnädelbach, and K. Glover, "Using Adaptive Architecture to Probe Attitudes Towards Ubiquitous Monitoring," in IEEE International Symposium on Technology and Society, 2013, p. In Press. 